\title{
MENSURAÇÃO DA EFICIÊNCIA DO DNIT NA MANUTENÇÃO DA MALHA RODOVIÁRIA NACIONAL
}

\section{ARTIGO ORIGINAL}

SOARES, Carlos Henrique ${ }^{1}$

SOARES, Carlos Henrique. Mensuração da eficiência do DNIT na manutenção da malha rodoviária nacional. Revista Científica Multidisciplinar Núcleo do Conhecimento. Ano 05, Ed. 08, Vol. 14, pp. 64-82. Agosto de 2020. ISSN: 2448-0959, Link

acesso: https://www.nucleodoconhecimento.com.br/administracao/mensuracao-da-

eficiencia

\section{RESUMO}

Com a reforma administrativa de 1988, o aparelho do Estado passa a dar importância, principalmente, a dois diferentes aspectos: a qualidade na prestação do serviço público e a cultura gerencial nas organizações. Com o intuito de informar sobre a eficiência ou ineficiência na Administração Pública o presente artigo traz a mensuração quantitativa da eficiência na execução orçamentária e financeira das despesas com a manutenção da malha rodoviária federal por meio da Análise Envoltória de Dados (DEA-BCC-O) onde evidenciou-se o nível de eficiência dos gastos públicos realizados em Unidades Tomadoras de Decisão do DNIT servindo de base para que seus gestores tomem decisões no sentido de apresentarem resultados mais adequados às necessidades sociais.

Palavras-Chave: Mensuração, eficiência, DEA.

\footnotetext{
${ }^{1}$ Mestre em Administração Pública pela Universidade Federal do Mato Grosso do Sul (UFMS).
} 


\section{INTRODUÇÃO}

A partir da Emenda Constitucional № 19 de 1998 (Reforma Administrativa) a administração gerencial no setor público tem como objetivo superar a falta de eficiência em atender as novas demandas do mundo globalizado. Este novo modelo gerencial estabelece como diretriz para os gestores públicos a busca da eficiência da Administração Pública como forma de ampliar os benefícios à sociedade. Diante desse novo contexto, a questão do uso eficiente de recursos públicos tem ganhado importância nas discussões referentes às boas práticas de gestão. Assim, uma gestão pública eficiente se torna essencial para a garantia da qualidade na prestação de serviços governamentais, além de garantir a utilização racional dos recursos públicos.

Em 2007, o governo federal instituiu o Programa de Aceleração do Crescimento (PAC), que é constituído de medidas de estímulo ao investimento privado, de ampliação dos investimentos públicos em infraestrutura e voltadas à melhoria da qualidade do gasto público e ao controle da expansão dos gastos correntes no âmbito da Administração Pública Federal. Com relação às rodovias, o PAC tem como objetivo a expansão e modernização da malha rodoviária nacional a fim de promover a segurança viária, a redução dos custos logísticos para gerar um potencial multiplicador sobre ampla gama de setores produtivos.

No âmbito da Administração Pública Federal, o DNIT foi inserido no PAC, principalmente, no eixo transportes, com a missão de expansão do sistema rodoviário brasileiro, sua manutenção, segurança rodoviária, estudos e projetos a fim de se buscar aumentar a competitividade do Brasil. Para isso, a autarquia ficou responsável pela execução orçamentária e financeira de $37 \%$ dos recursos referentes ao PAC no período compreendido entre os anos de 2009 e 2015.

A relevância do presente estudo pode ser percebida na busca de resposta à pergunta: A execução orçamentária e financeira do PAC para a manutenção da malha rodoviária de responsabilidade do DNIT foi realizada de forma eficiente? Para a avaliação da eficiência do DNIT na aplicação dos recursos públicos, o método DEA é apontado 
como um dos mais indicados para o setor público, pois transforma, em um único valor, múltiplos outputs e múltiplos inputs de diferentes Unidades (DMU). Nesse artigo, foi utilizado o método indutivo-comparativo com mensuração quantitativa para explicar cientificamente o fenômeno da eficiência.

A mensuração quantitativa foi realizada por meio da Análise Envoltória de Dados (DEA) no modelo BCC orientado a outputs. (BCC-O) e as constatações desta pesquisa evidenciam o nível de eficiência dos gastos públicos realizados em Unidades Tomadoras de Decisão do DNIT e dessa forma, serve de base para que gestores tomem decisões no sentido de apresentarem resultados mais adequados às necessidades sociais.

\section{EFICIÊNCIA NA ADMINISTRAÇÃO PÚBLICA}

De acordo com Paludo (2014), com a Emenda Constitucional no 19 de 1998 (Reforma Administrativa) o aparelho do Estado passa a dar importância, principalmente, a dois diferentes aspectos: a qualidade na prestação do serviço público e a cultura gerencial nas organizações públicas do país. Com isso, fica estabelecido que as Administrações Públicas Direta e Indireta devem obedecer ao princípio da eficiência e, em consequência disso, os gestores públicos devem buscar a eficiência da Administração Pública que tem como beneficiário a sociedade. Uma gestão pública eficiente se torna essencial, tendo em vista que representa a necessidade de reduzir custos e aumentar a qualidade dos serviços.

O conceito de eficiência, segundo Rutgers; Meer (2010, apud DA SILVA, 2015), está interligado a outros importantes conceitos como eficácia, efetividade, insumo, produto e resultados, conforme a figura: 
Figura 01 - Eficiência em relação a outros conceitos

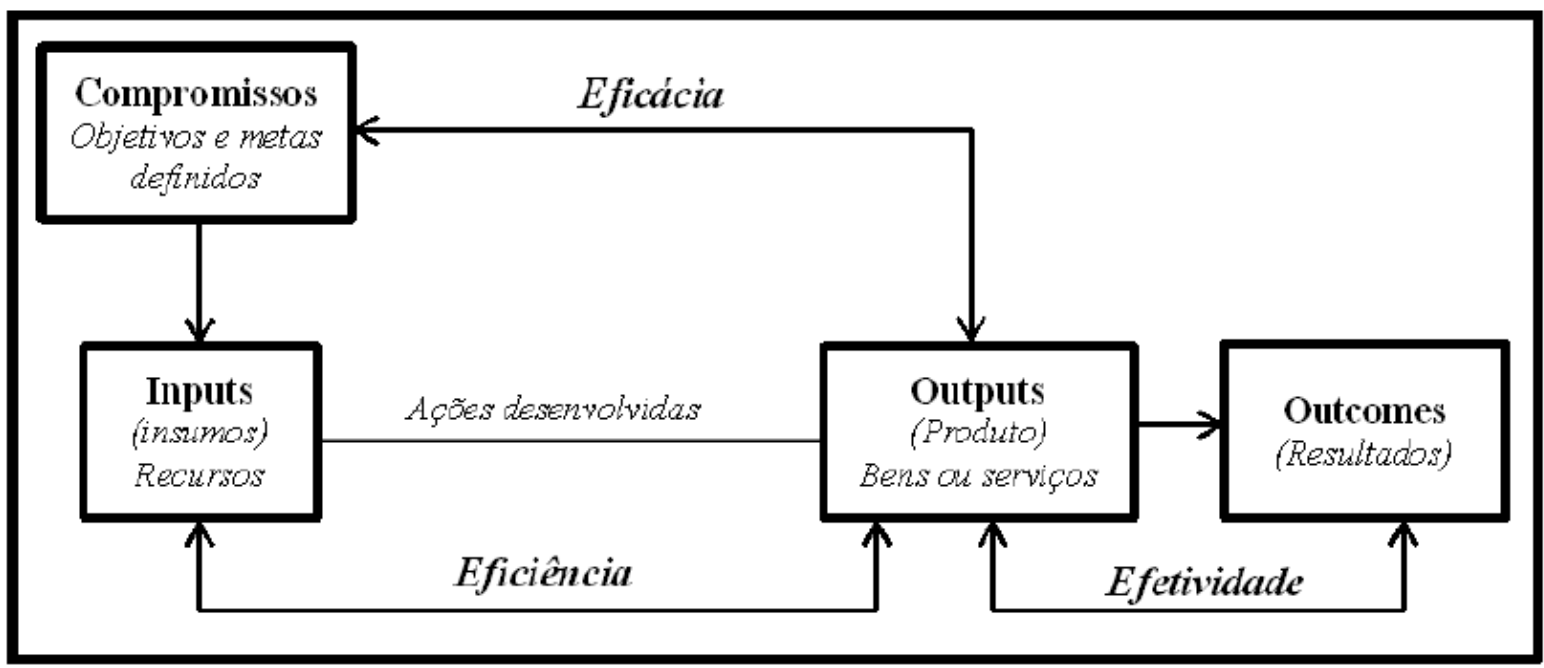

Fonte: Da Silva (2015)

Dessa forma, Rutgers; Meer (2010, apud DA SILVA, 2015) argumentam que enquanto o conceito de eficiência se refere à relação entre o montante de recursos (inputs) e produtos/serviços (outputs), o conceito de eficácia está ligado ao alcance de determinadas metas/objetivos sem considerar os custos implicados. Já a efetividade, segundo os autores, indica os trabalhos realmente executados e seus resultados (outcomes).

No caso da Administração Pública, a eficiência deve ser considerada sob a perspectiva da relação entre inputs e outputs. Nesse sentido, tendo em vista essa relação, Wilson (1989, apud DA SILVA 2015) ao comparar os setores públicos e privados, argumenta que a Administração Pública enfrenta dificuldades para atingir maiores níveis de eficiência. Isso por quê:

[...] enquanto as organizações privadas possuem apenas um output estabelecido, lucro ou receita, a Administração Pública depara-se com múltiplos outputs a serem alcançados, incluindo a necessidade de manter a reputação de integridade, a confiança da sociedade e o apoio de grupos importantes. Nesse contexto, outros valores acabam tendo preponderância em relação à eficiência, ou seja, nem sempre este conceito torna-se o objetivo mais importante para o setor público. (DA SILVA, 2015).

Disponível em: https://www.nucleodoconhecimento.com.br/administracao/mensuracao-daeficiencia 
Dessa forma, segundo Da Silva (2015), torna-se evidente que a Administração Pública tenha resultados menos eficiente que no setor privado, tendo em vista os inúmeros outputs perseguidos por ela.

São inúmeras as situações e dificuldades enfrentadas que reduzem o nível de eficiência do setor público, como questões culturais, desigualdades sociais, bem como a rigidez legal e o controle incidente sobre a utilização dos recursos públicos. Nesse sentido, Wilson (1989, apud DA SILVA, 2015), aponta que essa redução no nível de eficiência do setor público, também, é devido a necessidade de integridade fiscal, equidade e responsabilidade na prestação de contas à sociedade. Essas limitações, segundo o autor, encarecem o bem/serviço e/ou retardam o seu fornecimento, tornando a Administração Pública menos eficiente.

\section{MENSURAÇÃO DA EFICIÊNCIA}

As informações sobre a eficiência ou ineficiência na Administração Pública serão mais significativas se suas potenciais causas puderem ser avaliadas e dessa forma, auxiliarem os gestores públicos e a sociedade na verificação dos resultados esperados. Mensurar a eficiência é, portanto, um processo de grande importância no âmbito da Administração Pública.

Segundo De Lima; Diniz (2013), a mensuração da eficiência da prestação de serviços público tem aumentado cada vez mais e isso é devido a cobrança, por parte da sociedade, por mais transparência nos resultados de políticas públicas. Com isso, o planejamento e a gestão da máquina pública passaram a utilizar indicadores de desempenho (NOGUEIRA, 2010, apud DE LIMA; DINIZ, 2013).

No entanto, Arretche (1998) ensina que a eficiência no setor público não é simplesmente uma equação entre custos reduzidos e os melhores resultados (mensuráveis) possíveis. A utilização de indicadores não elimina a necessidade de planejar e aplicar uma política com maior eficiência possível, eliminando custos desnecessários e não condizentes com o princípio da equidade (ARRETCHE, 1998). 
De acordo com Camargo; Guimarães (2013) o Estado deve buscar o planejamento, desenvolvimento e execução de suas funções de forma eficaz e efetiva, utilizando-se de novas técnicas para se buscar satisfatórios para a sociedade. No entanto, sem o comprometimento por parte dos gestores e agentes públicos esses resultados não serão alcançados (CAMARGO; GUIMARÃES, 2013), ou seja, existe uma demanda na medição da eficiência dos serviços públicos a partir dos gestores das organizações públicas.

Nesse sentido, Arretche (1998) entende que a avaliação da eficiência deva ser necessariamente desenvolvida no setor público. Segundo a autora, a escassez de recursos exige uma maior racionalização do gasto público. Com isso, avaliar a eficiência do Setor Público tornam-se cada vez mais necessária, pois em regimes democráticos, como no Brasil, o uso de recursos públicos de forma eficiente e transparente constitui uma das condições para a confiança pública no governo (ARRETCHE, 1998).

\section{MÉTODO DEA (DATA ENVELOPMENT ANALYSIS)}

De acordo com a literatura existente, o método DEA é apontado como um dos mais indicados para o setor público e, portanto, será o método selecionado para mensurar e avaliar a eficiência do DNIT na execução orçamentária e financeira do PAC para a manutenção da malha rodoviária federal, dadas as suas vantagens.

O método não paramétrico por Análise Envoltória de Dados (DEA) é uma técnica de programação matemática desenvolvida por Charnes; Cooper; Rhodes (1978) que permite avaliar o grau de eficiência produtiva entre Unidades Tomadoras de Decisão (DMU - Decision Making Units), considerando os recursos de que dispõe (inputs) para se alcançar resultados (outputs). O princípio básico da DEA é medir e comparar o desenvolvimento das unidades tomadoras de decisão, que realizam tarefas similares, considerando a relação inputs/outputs. Dessa forma, a DEA permite otimizar individualmente cada uma das observações, uma em relação as demais, formando assim uma fronteira de eficiência (melhor relação entre insumos e produtos). 
Segundo Peña (2008), a característica essencial do DEA é a transformação dos múltiplos outputs e dos múltiplos inputs das DMU's em um único valor para output e input (output virtual e input virtual), respectivamente, para cada uma das DMU's. A razão entre os outputs e inputs virtuais irá provê uma medida relativa para a eficiência técnica (PEÑA, 2008).

\section{MENSURAÇÃO DA EFICIÊNCIA DO DNIT NA MANUTENÇÃO DA MALHA RODOVIÁRIA NACIONAL}

De acordo com os relatórios PAC da Secretaria de Orçamento Federal do MPOG[2], no período compreendido entre os anos de 2009 a 2015, o DNIT é responsável pela execução orçamentária e financeira de $31 \%$ dos recursos destinados ao PAC e desse percentual, 37\% foram destinados à Manutenção da Malha Rodoviária Federal, conforme o quadro abaixo:

Tabela 01 - Execução da despesa orçamentária para a manutenção da malha rodoviária federal a cargo do DNIT - despesa (exercício financeiro).

\begin{tabular}{l|l|l|l|l|}
\multicolumn{2}{c}{ R 1,00 (valores correntes) } & \multicolumn{3}{l}{} \\
SR/DNIT & $\begin{array}{l}\text { Dotação } \\
\text { Autorizada }\end{array}$ & $\begin{array}{l}\text { Valores } \\
\text { Empenhados }\end{array}$ & $\begin{array}{l}\text { Valores } \\
\text { Liquidados }\end{array}$ & Valores \\
& Pagos \\
\hline AM/RR (01) & 2.101 .613 .153 & 1.885 .274 .062 & 1.016 .504 .081 & 335.601 .644 \\
\hline PA/AP (02) & 1.726 .962 .191 & 1.614 .953 .237 & 979.303 .287 & 441.419 .467 \\
\hline CE (03) & 1.096 .962 .158 & 1.067 .830 .084 & 476.684 .858 & 224.957 .160 \\
\hline PE (04) & 1.293 .664 .148 & 1.223 .847 .518 & 739.168 .798 & 260.500 .577 \\
\hline BA (05) & 2.638 .700 .600 & 2.036 .035 .136 & 1.268 .669 .868 & 610.296 .715 \\
\hline MG (06) & 5.755 .923 .887 & 5.563 .967 .154 & 3.572 .738 .608 & 2.308 .241 .909 \\
\hline RJ (07) & 823.882 .350 & 802.672 .977 & 485.038 .596 & 239.013 .259 \\
\hline SP (08) & 161.433 .498 & 134.487 .942 & 93.220 .961 & 35.125 .465 \\
\hline PR (09) & 1.977 .352 .015 & 1.567 .756 .945 & 1.009 .629 .184 & 431.316 .258
\end{tabular}




\begin{tabular}{|l|l|l|l|l|}
\hline RS (10) & 2.138 .791 .902 & 2.105 .062 .305 & 1.401 .607 .989 & 667.162 .682 \\
\hline MT (11) & 2.119 .881 .532 & 2.101 .582 .294 & 1.261 .286 .883 & 671.314 .540 \\
\hline GO/DF (12) & 2.072 .423 .893 & 1.979 .073 .852 & 1.398 .072 .440 & 802.580 .233 \\
\hline PB (13) & 683.905 .455 & 645.107 .294 & 402.018 .974 & 227.243 .135 \\
\hline RN (14) & 647.123 .178 & 611.511 .853 & 352.707 .886 & 153.254 .771 \\
\hline MA (15) & 2.279 .991 .728 & 2.248 .246 .610 & 1.294 .526 .385 & 866.889 .265 \\
\hline SC (16) & 873.944 .653 & 807.958 .996 & 465.432 .182 & 145.165 .042 \\
\hline ES (17) & 477.006 .191 & 464.739 .381 & 302.701 .178 & 153.719 .021 \\
\hline PI (18) & 873.898 .392 & 813.403 .213 & 410.437 .430 & 176.417 .327 \\
\hline MS (19) & 2.357 .522 .517 & 1.993 .011 .387 & 1.321 .254 .945 & 786.177 .553 \\
\hline AL (20) & 405.137 .543 & 391.881 .586 & 205.768 .246 & 108.025 .213 \\
\hline SE (21) & 158.732 .665 & 145.720 .990 & 89.300 .166 & 23.239 .079 \\
\hline RO/AC (22) & 1.942 .510 .321 & 1.888 .675 .328 & 1.234 .678 .886 & 658.248 .268 \\
\hline TO (23) & 1.320 .708 .150 & 908.676 .315 & 572.940 .971 & 293.367 .203 \\
\hline TOTAIS & 35.928 .072 .120 & 33.001 .476 .459 & 20.353 .692 .802 & 10.619 .275 .786 \\
\hline
\end{tabular}

Fonte: dados da pesquisa.

Com relação aos Restos a Pagar, no período compreendido entre os anos de 2009 a 2015, os relatórios PAC da Secretaria de Orçamento Federal do MPOG apresentaram os dados a seguir:

Tabela 02 - Execução da despesa orçamentária para a manutenção da malha rodoviária federal a cargo do DNIT - Restos a pagar.

$\mathrm{R} \$ 1,00$ (valores correntes)

\begin{tabular}{|l|l|l|l|}
\hline SR/DNIT & Valores Inscritos & $\begin{array}{l}\text { Valores } \\
\text { Cancelados }\end{array}$ & Valores Pagos \\
\hline AM/RR (01) & 2.345 .309 .101 & 180.040 .776 & 833.405 .044 \\
\hline PA/AP (02) & 1.374 .597 .192 & 67.200 .869 & 764.326 .167 \\
\hline CE (03) & 1.220 .887 .333 & 63.990 .566 & 610.380 .301 \\
\hline
\end{tabular}


https://www.nucleodoconhecimento.com.br

\begin{tabular}{|l|l|l|l|}
\hline PE (04) & 1.026 .504 .497 & 89.116 .727 & 619.183 .099 \\
\hline BA (05) & 1.838 .173 .049 & 167.661 .960 & 1.086 .423 .049 \\
\hline MG (06) & 3.974 .059 .421 & 336.248 .788 & 2.371 .970 .909 \\
\hline RJ (07) & 809.534 .305 & 75.428 .450 & 393.840 .218 \\
\hline SP (08) & 125.193 .640 & 14.874 .496 & 53.887 .550 \\
\hline PR (09) & 1.308 .995 .256 & 73.905 .354 & 751.582 .322 \\
\hline RS (10) & 1.840 .281 .137 & 167.406 .254 & 1.024 .978 .274 \\
\hline MT (11) & 2.010 .695 .938 & 162.881 .898 & 1.142 .118 .496 \\
\hline GO/DF (12) & 1.745 .038 .461 & 151.549 .331 & 925.989 .149 \\
\hline PB (13) & 456.847 .531 & 13.115 .001 & 329.722 .407 \\
\hline RN (14) & 555.384 .092 & 65.189 .300 & 244.257 .950 \\
\hline MA (15) & 1.655 .102 .199 & 72.326 .093 & 1.001 .849 .640 \\
\hline SC (16) & 1.074 .135 .457 & 78.988 .528 & 390.653 .965 \\
\hline ES (17) & 387.065 .928 & 26.084 .811 & 234.611 .860 \\
\hline PI (18) & 876.824 .022 & 65.216 .752 & 493.196 .345 \\
\hline MS (19) & 1.469 .037 .791 & 203.029 .749 & 878.478 .590 \\
\hline AL (20) & 426.220 .959 & 21.825 .227 & 180.890 .451 \\
\hline SE (21) & 165.059 .704 & 21.720 .340 & 87.400 .636 \\
\hline RO/AC (22) & 1.433 .969 .943 & 186.079 .906 & 704.488 .758 \\
\hline TO (23) & 674.799 .264 & 80.747 .713 & 421.028 .995 \\
\hline TOTAIS & 28.793 .716 .220 & 2.384 .628 .889 & 15.544 .664 .175 \\
\hline & & & \\
\hline
\end{tabular}

Fonte: dados da pesquisa.

Para avaliar a eficiência na execução orçamentária e financeira dos recursos destinados ao PAC para a manutenção da malha rodoviária de responsabilidade do DNIT, foi utilizado o método indutivo-comparativo com mensuração quantitativa para explicar cientificamente o fenômeno da eficiência na execução orçamentária e financeira das despesas com a manutenção da malha rodoviária federal. A mensuração quantitativa foi realizada com a Análise Envoltória de Dados (DEA) e para

Disponível em: https://www.nucleodoconhecimento.com.br/administracao/mensuracao-daeficiencia 
isso foi realizado o software SIAD v.3.0 - Sistema Integrado de Apoio à Decisão v.3.0. (MEZA et al., 2011) no modelo BCC com orientação a outputs. (BCC-O).

A população da pesquisa foi do tipo intencional e não probabilística e compreendeu as unidades regionais pertencentes à estrutura do DNIT que representam as Unidades Tomadoras de Decisão (DMUs). De acordo com Peña (2008), as DMU's devem produzir os mesmos bens ou serviços e ainda utilizar os mesmos insumos e segundo Faria; Januzzi; Silva (2008), deve-se garantir que os indicadores relativos às DMU's sejam confiáveis, uma vez que o método DEA é sensível a valores extremos, o que Ihe confere, ao mesmo tempo, uma limitação com relação a dados imprecisos e uma potencialidade quando da existência de valores extremos que representem verdadeiramente uma situação de boas práticas.

Diante disso, foram selecionadas como DMU's as 23 Superintendências Regionais do DNIT. Dessa forma, é possível atender ao quesito da homogeneidade das unidades ao considerarmos que essas superintendências utilizam o mesmo input para produzem os mesmos outputs e que estão inseridas no mesmo contexto organizacional.

Quanto ao input e aos outputs, os dados referentes a execução orçamentária e financeira dos recursos do PAC destinados a manutenção da malha rodoviária de responsabilidade do DNIT foram coletados da base de dados do Portal do Orçamento Federal (MPOG). Foram considerados como input, os valores orçamentários autorizados para o provimento dos respectivos outputs, ou seja, a dotação autorizada pela LOA. Já com relação aos outputs, considerou-se os índices de desempenho apresentados nos relatórios mensais do PAC elaborados pela Secretaria de Orçamento Federal[3].

Quanto ao período da pesquisa, foram considerados os dados referentes ao período compreendido entre os anos de 2009 e 2015, tendo em vista que os dados relativos ao PAC dos anos de 2007 e 2008 não apresentam informações suficientes para a apuração da execução orçamentária e financeira de competência do DNIT. 
Portanto, as variáveis escolhidas para a Análise Envoltória de Dados (DEA) são as seguintes: INPUT: Dotação autorizada pela LOA e OUTPUTs: (1) Percentual da despesa empenhada em relação à dotação autorizada; (2) Percentual da despesa paga dentro do exercício financeiro em relação a despesa liquidada dentro do exercício financeiro e (3) Percentual de pagamentos de restos a pagar em relação às Inscrições e cancelamentos dos mesmos, conforme a tabela abaixo:

Tabela 03- Variáveis da pesquisa

\begin{tabular}{|c|c|c|c|c|}
\hline DMUs & \multicolumn{4}{|l|}{23 SR/DNIT } \\
\hline INPUT & \multicolumn{4}{|c|}{ Dotação autorizada pela LOA } \\
\hline \multirow{3}{*}{ OUTPUTS } & \multicolumn{4}{|c|}{ (1) Valores Empenhados/Dotação autorizada (\%) } \\
\hline & \multicolumn{4}{|c|}{ (2) Valores Pagos/Valores Liquidados (\%) } \\
\hline & \multicolumn{4}{|c|}{$\begin{array}{l}\text { (3) Valores de RP pagos/Valores de RP inscritos menos os valores } \\
\text { de RP cancelados (\%) }\end{array}$} \\
\hline DMUs & INPUT & OUTPUT 1 & OUTPUT 2 & $\begin{array}{l}\text { OUTPUT } \\
3\end{array}$ \\
\hline DMU_1 (AM/RR) & $\begin{array}{l}\mathrm{R} \$ \\
2.101 .613 .153,00\end{array}$ & $89,706 \%$ & $33,015 \%$ & $38,490 \%$ \\
\hline DMU_2 (AP/PA) & $\begin{array}{l}\mathrm{R} \$ \\
1.726 .962 .191,00\end{array}$ & $93,514 \%$ & $45,075 \%$ & $58,462 \%$ \\
\hline DMU_3 (CE) & $\begin{array}{l}\mathrm{R} \$ \\
1.096 .962 .158,00\end{array}$ & $97,344 \%$ & $47,192 \%$ & $52,760 \%$ \\
\hline DMU_4 (PE) & $\begin{array}{l}\mathrm{R} \$ \\
1.293 .664 .148,00\end{array}$ & $94,603 \%$ & $35,242 \%$ & $66,054 \%$ \\
\hline DMU_5 (BA) & $\begin{array}{l}\mathrm{R} \$ \\
2.638 .700 .600,00\end{array}$ & $77,161 \%$ & $48,105 \%$ & $65,035 \%$ \\
\hline
\end{tabular}

Disponível em: https://www.nucleodoconhecimento.com.br/administracao/mensuracao-daeficiencia 


\begin{tabular}{|c|c|c|c|c|}
\hline DMU_6 (MG) & $\begin{array}{l}\mathrm{R} \$ \\
5.755 .923 .887,00\end{array}$ & $96,665 \%$ & $64,607 \%$ & $65,203 \%$ \\
\hline DMU_7 (RJ) & $\mathrm{R} \$ 823.882 .350,00$ & $97,426 \%$ & $49,277 \%$ & $53,649 \%$ \\
\hline DMU_8 (SP) & $\mathrm{R} \$ 161.433 .498,00$ & $83,309 \%$ & $37,680 \%$ & $48,847 \%$ \\
\hline DMU_9 (PR) & $\begin{array}{l}\mathrm{R} \$ \\
1.977 .352 .015,00\end{array}$ & $79,286 \%$ & $42,720 \%$ & $60,852 \%$ \\
\hline DMU_10 (RS) & $\begin{array}{l}\mathrm{R} \$ \\
2.138 .791 .902,00\end{array}$ & $98,423 \%$ & $47,600 \%$ & $61,270 \%$ \\
\hline DMU_11 (MT) & $\begin{array}{l}R \$ \\
2.119 .881 .532,00\end{array}$ & $99,137 \%$ & $53,225 \%$ & $61,809 \%$ \\
\hline DMU_12 (GO/DF) & $\begin{array}{l}\mathrm{R} \$ \\
2.072 .423 .893,00\end{array}$ & $95,496 \%$ & $57,406 \%$ & $58,111 \%$ \\
\hline DMU_13 (PB) & $\mathrm{R} \$ 683.905 .455,00$ & $94,327 \%$ & $56,525 \%$ & $74,307 \%$ \\
\hline DMU_14 (RN) & $\mathrm{R} \$ 647.123 .178,00$ & $94,497 \%$ & $43,451 \%$ & $49,829 \%$ \\
\hline DMU_15 (MA) & $\begin{array}{l}R \$ \\
2.279 .991 .728,00\end{array}$ & $98,608 \%$ & $66,966 \%$ & $63,297 \%$ \\
\hline DMU_16 (SC) & $\mathrm{R} \$ 873.944 .653,00$ & $92,450 \%$ & $31,189 \%$ & $39,256 \%$ \\
\hline DMU_17 (ES) & $\mathrm{R} \$ 477.006 .191,00$ & $97,428 \%$ & $50,782 \%$ & $64,993 \%$ \\
\hline DMU_18 (PI) & $\mathrm{R} \$ 873.898 .392,00$ & $93,078 \%$ & $42,983 \%$ & $60,768 \%$ \\
\hline DMU_19 (MS) & $\begin{array}{l}\mathrm{R} \$ \\
2.357 .522 .517,00\end{array}$ & $84,538 \%$ & $59,502 \%$ & $69,390 \%$ \\
\hline DMU_20 (AL) & $\mathrm{R} \$ 405.137 .543,00$ & $96,728 \%$ & $52,498 \%$ & $44,731 \%$ \\
\hline DMU_21 (SE) & $\mathrm{R} \$ 158.732 .665,00$ & $91,803 \%$ & $26,024 \%$ & $60,975 \%$ \\
\hline DMU_22 (AC/RO) & $\begin{array}{l}\mathrm{R} \$ \\
1.942 .510 .321,00\end{array}$ & $97,229 \%$ & $53,313 \%$ & $56,454 \%$ \\
\hline DMU_23 (TO) & $\begin{array}{l}\mathrm{R} \$ \\
1.320 .708 .150,00\end{array}$ & $68,802 \%$ & $51,204 \%$ & $70,874 \%$ \\
\hline
\end{tabular}

Fonte: dados da pesquisa.

Disponível em: https://www.nucleodoconhecimento.com.br/administracao/mensuracao-daeficiencia 
Segundo Wilson (1989, apud DA SILVA, 2015), a redução dos custos não significa o mesmo que aumentar a eficiência. As restrições orçamentárias podem comprometer o nível de produção, tornando-a ineficiente e extinguindo a flexibilidade gerencial frente a circunstâncias inesperadas. Assim, a redução orçamentária tem como consequência a diminuição do volume de bens/serviços a serem adquiridos e dessa forma, produz um efeito de aumento do custo unitário do bem/serviço, uma vez que os fornecedores deixam de se beneficiar da economia advinda da produção de larga escala. Nesse sentido, com a utilização do software SIAD v.3.0 (MEZA et al., 2011), aplicou-se o modelo BCC, com orientação output, isto é, visou-se maximizar os outputs sem diminuir o input.

\subsection{ANÁLISE DOS RESULTADOS}

$\mathrm{Na}$ Tabela 01 são apresentados os resultados completos das 23 DMUs, contendo os escores de eficiência das fronteiras padrão, invertida, composta e composta normalizada, respectivamente, de acordo com o software SIAD v.3.0.

Tabela 04- Fronteiras de eficiência

\begin{tabular}{|c|c|c|c|c|}
\hline DMU & Padrão & Invertida & Composta & Composta* \\
\hline DMU_6 (MG) & $100 \%$ & $100 \%$ & $50 \%$ & $84 \%$ \\
\hline DMU_8 (SP) & $100 \%$ & $100 \%$ & $50 \%$ & $84 \%$ \\
\hline DMU_11 (MT) & $100 \%$ & $83 \%$ & $58 \%$ & $98 \%$ \\
\hline DMU_13 (PB) & $100 \%$ & $81 \%$ & $59 \%$ & $100 \%$ \\
\hline DMU_15 (MA) & $100 \%$ & $84 \%$ & $58 \%$ & $98 \%$ \\
\hline DMU_17 (ES) & $100 \%$ & $82 \%$ & $59 \%$ & $99 \%$ \\
\hline DMU_20 (AL) & $100 \%$ & $100 \%$ & $50 \%$ & $84 \%$ \\
\hline DMU_21 (SE) & $100 \%$ & $100 \%$ & $50 \%$ & $84 \%$ \\
\hline DMU_7 (RJ) & $100 \%$ & $87 \%$ & $56 \%$ & $95 \%$ \\
\hline DMU_10 (RS) & $99 \%$ & $84 \%$ & $58 \%$ & $97 \%$ \\
\hline DMU_3 (CE) & $99 \%$ & $87 \%$ & $56 \%$ & $94 \%$ \\
\hline DMU_19 (MS) & $99 \%$ & $91 \%$ & $54 \%$ & $91 \%$ \\
\hline
\end{tabular}

Disponível em: https://www.nucleodoconhecimento.com.br/administracao/mensuracao-daeficiencia 


\begin{tabular}{|l|l|l|l|l|}
\hline DMU_22 (AC/RO) & $98 \%$ & $86 \%$ & $56 \%$ & $95 \%$ \\
\hline DMU_4 (PE) & $98 \%$ & $93 \%$ & $52 \%$ & $88 \%$ \\
\hline DMU_23 (TO) & $97 \%$ & $100 \%$ & $48 \%$ & $82 \%$ \\
\hline DMU_14 (RN) & $97 \%$ & $91 \%$ & $53 \%$ & $89 \%$ \\
\hline DMU_12 (GO/DF) & $97 \%$ & $87 \%$ & $55 \%$ & $93 \%$ \\
\hline DMU_18 (PI) & $95 \%$ & $89 \%$ & $53 \%$ & $90 \%$ \\
\hline DMU_2 (AP/PA) & $95 \%$ & $88 \%$ & $54 \%$ & $90 \%$ \\
\hline DMU_16 (SC) & $94 \%$ & $100 \%$ & $47 \%$ & $80 \%$ \\
\hline DMU_5 (BA) & $92 \%$ & $100 \%$ & $46 \%$ & $77 \%$ \\
\hline DMU_1 (AM/RR) & $91 \%$ & $100 \%$ & $45 \%$ & $76 \%$ \\
\hline DMU_9 (PR) & $85 \%$ & $100 \%$ & $42 \%$ & $71 \%$ \\
\hline
\end{tabular}

Fonte: dados da pesquisa

Com base na tabela de fronteiras de eficiência, é possível avaliar comparativamente a eficiência das Superintendências Regionais do DNIT na execução orçamentária e financeira dos recursos destinados ao PAC no período entre 2009 a 2015 e como consequência, identificar as Superintendências Regionais que apresentaram os melhores e piores níveis de eficiência na execução desses recursos.

De acordo com os escores de eficiência apresentados na coluna referente à fronteira padrão fica evidenciado que a fronteira de eficiência na aplicação de recursos destinados ao PAC para a manutenção da malha rodoviária federal é composta pelas seguintes Superintendências Regionais: SR/MG, SR/SP, SR/RS, SR/MT, SR/GO-DF, SR/PB, SR/MA, SR/ES, SR/AL, SR/SE e SR/RJ.

No entanto, com base nos fundamentos teóricos do modelo DEA empregado, observa-se que a fronteira padrão não permite identificar, no contexto analisado, qual foi a superintendência mais eficiente, o que impossibilitaria alcançar um dos objetivos específicos que é a construção de um ranking de eficiência relativa das superintendências regionais do DNIT, pois, para tanto, seria necessário fazer o desempate entre as superintendências consideradas eficientes. Nesse caso, de

Disponível em: https://www.nucleodoconhecimento.com.br/administracao/mensuracao-daeficiencia 
acordo com Soares de Melo et. al. (2005), existe a possibilidade do uso da fronteira de eficiência invertida para identificar as DMUs que inicialmente são apresentadas como eficientes. De acordo com Pimenta; Soares de Melo (2005), a utilização da fronteira invertida permite a avaliação das DMUs naquilo em que elas são ineficientes.

Dessa forma, realizou-se a discriminação da eficiência com base na aplicação da fronteira invertida, pois todas as DMUs eficientes na fronteira padrão teoricamente passariam ao status de ineficientes na segunda fronteira e vice-versa. No entanto, a fronteira invertida pode caracterizar uma falsa eficiência, pois permite a identificação de DMUs que são avaliadas como eficientes através da fronteira padrão e consideradas ineficientes através da fronteira invertida (PIMENTA; SOARES DE MELO, 2005). Diante disso, para a construção do ranking, calculou-se, então, a fronteira composta normalizada ou Eficiência Normalizada:

Tabela 05- Eficiências normalizadas

\begin{tabular}{|l|l|l|}
\hline Ranking & DMU & Eficiência Normalizada \\
\hline 1 & DMU_13 (PB) & $100 \%$ \\
\hline 2 & DMU_17 (ES) & $99 \%$ \\
\hline 3 & DMU_11 (MT) & $98 \%$ \\
\hline 4 & DMU_15 (MA) & $98 \%$ \\
\hline 5 & DMU_10 (RS) & $97 \%$ \\
\hline 6 & DMU_7 (RJ) & $95 \%$ \\
\hline 7 & DMU_22 (AC/RO) & $95 \%$ \\
\hline 8 & DMU_3 (CE) & $94 \%$ \\
\hline 9 & DMU_12 (GO/DF) & $93 \%$ \\
\hline 10 & DMU_19 (MS) & $91 \%$ \\
\hline 11 & DMU_2 (AP/PA) & $90 \%$ \\
\hline 12 & DMU_18 (PI) & $90 \%$ \\
\hline 13 & DMU_14 (RN) & $89 \%$ \\
\hline 14 & DMU_4 (PE) & $88 \%$ \\
\hline
\end{tabular}

Disponível em: https://www.nucleodoconhecimento.com.br/administracao/mensuracao-daeficiencia 


\begin{tabular}{|l|l|l|}
\hline 15 & DMU_6 (MG) & $84 \%$ \\
\hline 16 & DMU_8 (SP) & $84 \%$ \\
\hline 17 & DMU_20 (AL) & $84 \%$ \\
\hline 18 & DMU_21 (SE) & $84 \%$ \\
\hline 19 & DMU_23 (TO) & $82 \%$ \\
\hline 20 & DMU_16 (SC) & $80 \%$ \\
\hline 21 & DMU_5 (BA) & $77 \%$ \\
\hline 22 & DMU_1 (AM/RR) & $76 \%$ \\
\hline 23 & DMU_9 (PR) & $71 \%$ \\
\hline
\end{tabular}

Fonte: Dados da pesquisa

Com base nesse ranking, é possível afirmar que a SR/PB foi a mais eficiente na aplicação de recursos destinados ao PAC para a manutenção da malha rodoviária federal no período compreendido entre 2009 a 2015, seguida das SR/ES, SR/MT, SR/MA, SR/RS que atingiram grau de eficiência acima de $95 \%$.

Ressalta-se que a análise por meio da Eficiência Normalizada permitiu uma maior discriminação dos resultados inicialmente considerados eficientes, evidenciando, assim, cada uma das DMUs sob a forma de um ranking. Com isso, é possível, também, a identificação das superintendências piores colocadas no ranking de eficiência. Nesse caso, observa-se que a pior colocada foi a SR/PR com eficiência de $71 \%$.

\subsubsection{METAS (ALVOS) DE MELHORIA}

A identificação das metas ou alvos de melhoria permite que os gestores priorizem ações a serem tomadas a fim de que as Superintendências Regionais ineficientes alcançarem a eficiência na execução de recursos destinados ao PAC para a manutenção da malha rodoviária de responsabilidade do DNIT. Com base na análise de eficiência pela fronteira padrão (tabela 05), são identificadas as metas (alvos) de melhoria para as DMUs consideradas ineficientes. 


\subsubsection{OUTPUT 1}

Com relação aos valores empenhados conforme a dotação autorizada na LOA no período de 2009 a 2015 (output1), na tabela abaixo, são apresentadas as metas para as Superintendências com eficiência normalizada abaixo de $80 \%$ alcancem a eficiência nesse estágio da despesa, conforme a análise de eficiência padrão:

Tabela 6 - Metas para valores empenhados (\%) - SR abaixo de 80\%- output 1

\begin{tabular}{|l|l|l|l|}
\hline DMU & $\begin{array}{l}\text { Padrão } \\
(\%)\end{array}$ & Meta (Alvo) (\%) & Aumentar (\%) \\
\hline DMU_5 (BA) & 77,16 & 95,22 & 18,60 \\
\hline DMU_1 (AM/RR) & 89,70 & 99,11 & 9,41 \\
\hline DMU_9 (PR) & 79,28 & 94,92 & 15,64 \\
\hline
\end{tabular}

Fonte: Dados da pesquisa

\subsubsection{OUTPUT 2}

Com relação aos valores pagos conforme os valores liquidados no período de 2009 a 2015 (output 2), na tabela abaixo, são apresentadas as metas para as Superintendências com eficiência normalizada abaixo de $80 \%$ alcancem a eficiência nesse estágio da despesa, conforme a análise de eficiência padrão:

Tabela 7 - Metas para valores pagos (\%) - SR abaixo de $80 \%$ - output 2

\begin{tabular}{|l|l|l|l|}
\hline DMU & $\begin{array}{l}\text { Padrão } \\
(\%)\end{array}$ & Meta (Alvo) (\%) & Aumentar (\%) \\
\hline DMU_5 (BA) & 48,10 & 59,63 & 11,53 \\
\hline DMU_1 (AM/RR) & 33,01 & 53,19 & 20,18 \\
\hline DMU_9 (PR) & 42,72 & 58,58 & 15,86 \\
\hline
\end{tabular}

Fonte: Dados da pesquisa

Disponível em: https://www.nucleodoconhecimento.com.br/administracao/mensuracao-daeficiencia 


\subsubsection{OUTPUT 3}

Com relação ao percentual de pagamentos de restos a pagar conforme os valores inscritos em anos anteriores no período de 2009 a 2015 (output 3), na tabela abaixo, são apresentadas as metas para as Superintendências com eficiência normalizada abaixo de $80 \%$ alcancem a eficiência nesse estágio da despesa, conforme a análise de eficiência padrão:

Tabela 8 - Metas para valores empenhados (\%) - SR abaixo de $80 \%$ - output 3

\begin{tabular}{|l|l|l|l|}
\hline DMU & $\begin{array}{l}\text { Padrão } \\
(\%)\end{array}$ & Meta (Alvo) (\%) & Aumentar (\%) \\
\hline DMU_5 (BA) & 65,03 & 70,79 & 5,76 \\
\hline DMU_1 (AM/RR) & 38,49 & 61,84 & 23,35 \\
\hline DMU_9 (PR) & 60,85 & 71,98 & 11,13 \\
\hline
\end{tabular}

Fonte: Dados da pesquisa

\subsubsection{BENCHMARKS}

Diante desses resultados, é importante observar o que seria necessário para que essas superintendências consideradas ineficientes se tornem eficientes, isto é, para que estejam localizadas sobre a fronteira de eficiência.

Os benchmarks apresentam as projeções para que as superintendências que possuem algum tipo de ineficiência na execução orçamentária e financeira dos recursos destinados ao PAC se transformem em superintendências eficientes. As projeções são obtidas eliminando-se as ineficiências, ou seja, quanto de cada output deveria ser acrescido para que uma superintendência ineficiente se torne eficiente. Como instrumento de gestão, os benchmarks possibilitam a melhoria do desempenho das DMUs de forma comparativa, ou seja, uma superintendência não possui eficiência máxima somente se existir pelo menos outra superintendência, ou uma combinação de superintendências, que está utilizando de forma mais racional o input e produzindo,

Disponível em: https://www.nucleodoconhecimento.com.br/administracao/mensuracao-daeficiencia 
no mínimo, a mesma quantidade de outputs. No caso da orientação a outputs, foi considerado a maximização dos resultados sem diminuir os recursos.

Dentro desse contexto, a tabela abaixo apresenta os benchmarks, ou seja, as Superintendências Regionais que são reconhecidas como representantes das melhores práticas.

Tabela 9 - Benchmarks

\begin{tabular}{|c|c|c|c|c|c|c|c|c|}
\hline $\begin{array}{l}\quad \text { DMU } \\
\text { Analisa } \\
\text { da }\end{array}$ & $\begin{array}{l}\text { DMU_ } \\
6 \\
(\mathrm{MG})\end{array}$ & $\begin{array}{l}\text { DMU_ } \\
8 \text { (SP) }\end{array}$ & $\begin{array}{l}\text { DMU_ } \\
11 \\
(\mathrm{MT})\end{array}$ & $\begin{array}{l}\text { DMU_ } \\
13 \text { (PB) }\end{array}$ & $\begin{array}{l}\text { DMU_ } \\
15 \\
\text { (MA) }\end{array}$ & $\begin{array}{l}\text { DMU_ } \\
17 \text { (ES) }\end{array}$ & $\begin{array}{l}\text { DMU_ } \\
20(A L)\end{array}$ & $\begin{array}{l}\text { DMU_ } \\
21 \text { (SE) }\end{array}$ \\
\hline $\begin{array}{l}\text { DMU_1 } \\
\text { (AM/RR } \\
\text { ) }\end{array}$ & 0 & 0 & 0,98 & 0 & 0 & 0,01 & 0 & 0 \\
\hline $\begin{array}{l}\text { DMU_2 } \\
\text { (AP/PA) }\end{array}$ & 0 & 0 & 0,76 & 0 & 0 & 0,23 & 0 & 0 \\
\hline $\begin{array}{l}\text { DMU_3 } \\
\text { (CE) }\end{array}$ & 0 & 0 & 0,37 & 0 & 0 & 0,62 & 0 & 0 \\
\hline $\begin{array}{l}\text { DMU_4 } \\
\text { (PE) }\end{array}$ & 0 & 0 & 0,44 & 0,43 & 0 & 0,12 & 0 & 0 \\
\hline $\begin{array}{l}\text { DMU_5 } \\
\text { (BA) }\end{array}$ & 0,38 & 0 & 0 & 0,61 & 0 & 0 & 0 & 0 \\
\hline $\begin{array}{l}\text { DMU_6 } \\
\text { (MG) }\end{array}$ & 1 & 0 & 0 & 0 & 0 & 0 & 0 & 0 \\
\hline $\begin{array}{l}\text { DMU_7 } \\
\text { (RJ) }\end{array}$ & 0 & 0 & 0,21 & 0 & 0 & 0,78 & 0 & 0 \\
\hline $\begin{array}{l}\text { DMU_8 } \\
\text { (SP) }\end{array}$ & 0 & 1 & 0 & 0 & 0 & 0 & 0 & 0 \\
\hline $\begin{array}{l}\text { DMU_9 } \\
\text { (PR) }\end{array}$ & 0,25 & 0 & 0 & 0,74 & 0 & 0 & 0 & 0 \\
\hline
\end{tabular}




\begin{tabular}{|c|c|c|c|c|c|c|c|c|}
\hline $\begin{array}{l}\text { DMU_1 } \\
\text { 0 (RS) }\end{array}$ & 0 & 0 & 0,99 & 0 & 0 & 0 & 0 & 0 \\
\hline $\begin{array}{l}\text { DMU_1 } \\
1 \text { (MT) }\end{array}$ & 0 & 0 & 1 & 0 & 0 & 0 & 0 & 0 \\
\hline $\begin{array}{l}\text { DMU_1 } \\
2 \\
\text { (GO/DF } \\
\text { ) }\end{array}$ & 0 & 0 & 0,46 & 0 & 0,46 & 0,07 & 0 & 0 \\
\hline $\begin{array}{l}\text { DMU_1 } \\
3 \text { (PB) }\end{array}$ & 0 & 0 & 0 & 1 & 0 & 0 & 0 & 0 \\
\hline $\begin{array}{l}\text { DMU_1 } \\
4 \text { (RN) }\end{array}$ & 0 & 0 & 0,10 & 0 & 0 & 0,89 & 0 & 0 \\
\hline $\begin{array}{l}\text { DMU_1 } \\
5 \text { (MA) }\end{array}$ & 0 & 0 & 0 & 0 & 1 & 0 & 0 & 0 \\
\hline $\begin{array}{l}\text { DMU_1 } \\
6 \text { (SC) }\end{array}$ & 0 & 0 & 0,24 & 0 & 0 & 0,75 & 0 & 0 \\
\hline $\begin{array}{l}\text { DMU_1 } \\
7 \text { (ES) }\end{array}$ & 0 & 0 & 0 & 0 & 0 & 1 & 0 & 0 \\
\hline $\begin{array}{l}\text { DMU_1 } \\
8 \text { (PI) }\end{array}$ & 0 & 0 & 0,24 & 0 & 0 & 0,75 & 0 & 0 \\
\hline $\begin{array}{l}\text { DMU_1 } \\
9 \text { (MS) }\end{array}$ & 0,28 & 0 & 0 & 0,58 & 0,13 & 0 & 0 & 0 \\
\hline $\begin{array}{l}\text { DMU_2 } \\
0 \text { (AL) }\end{array}$ & 0 & 0 & 0 & 0 & 0 & 0 & 1 & 0 \\
\hline $\begin{array}{l}\text { DMU_2 } \\
1 \text { (SE) }\end{array}$ & 0 & 0 & 0 & 0 & 0 & 0 & 0 & 1 \\
\hline $\begin{array}{l}\text { DMU_2 } \\
2 \\
\text { (AC/RO } \\
\text { ) }\end{array}$ & 0 & 0 & 0,78 & 0 & 0,09 & 0,11 & 0 & 0 \\
\hline
\end{tabular}




\begin{tabular}{|l|l|l|l|l|l|l|l|l|}
\hline $\begin{array}{l}\text { DMU_2 } \\
\text { 3 (TO) }\end{array}$ & 0,12 & 0 & 0 & 0,87 & 0 & 0 & 0 & 0 \\
\hline
\end{tabular}

Fonte: Dados da pesquisa

A partir dos índices de eficiência calculados das 23 superintendências do DNIT consideradas para o cálculo da fronteira de eficiência (tabela 05), aproximadamente, $35 \%$ podem ser considerados como benchmarks, ou seja, servem de referência para as demais DMUs, pois se apresentam sobre a fronteira de eficiência com o indicador igual a 1 (um). Logo, representam as superintendências com melhor execução orçamentária e financeira na aplicação de recursos destinados ao PAC para a manutenção da malha rodoviária federal, pois conseguiram gerar os melhores resultados comparativos em relação às outras Unidades Tomadoras de Decisão avaliadas na pesquisa.

A Tabela a seguir apresenta as superintendências eficientes na execução orçamentária e financeira dos recursos do PAC para a manutenção da malha rodoviária de responsabilidade do DNIT no período compreendido entre 2009 a 2015, bem como o número de vezes que serviram de benchmark para as outras superintendências.

Tabela 10 - Quantidade de Benchmarks das DMUs eficientes

DMUs Eficientes

\begin{tabular}{|l|l|}
\hline DMU_6 (MG) & 4 \\
\hline DMU_8 (SP) & 0 \\
\hline DMU_11 (MT) & 11 \\
\hline DMU_13 (PB) & 5 \\
\hline DMU_15 (MA) & 3 \\
\hline DMU_17 (ES) & 10 \\
\hline DMU_20 (AL) & 0 \\
\hline DMU_21 (SE) & 0 \\
\hline
\end{tabular}

Disponível em: https://www.nucleodoconhecimento.com.br/administracao/mensuracao-da- 
Fonte: Dados da pesquisa

É possível observar que as Superintendências Regionais de Mato Grosso e de Espírito Santo são as que mais apareceram como benchmark para as demais superintendências. A SR/MT serve de referência para outras 11 DMUs, seguida da SR/ES que aparece como parâmetro de comparação para 10 DMUs. Dessa forma, a SR/MT foi a que apresentou melhores práticas, o que pode ser constatado através da posição dessa superintendência na fronteira de eficiência, pois atingiu um resultado acima da média no período compreendido entre 2009 a 2015, executando com eficiência os recursos do PAC para a manutenção da malha rodoviária de responsabilidade do DNIT quando comparada com as demais DMUs que possuem as variáveis estudadas em proporções similares.

Como na Tabela 05 foram apresentadas as DMUs que aparecem na fronteira de eficiência, as quais são referências para as demais, foi possível identificar, através dos resultados da DEA-BCC-O, quais são os benchmarks das superintendências menos eficientes, ou seja, ineficientes.

Tabela 11-Benchmarks das DMUs ineficientes

\begin{tabular}{|l|l|}
\hline DMU & BENCHMARKS \\
\hline DMU_1 (AM/RR) & DMU_11 (MT), DMU_17 (ES) \\
\hline DMU_2 (AP/PA) & DMU_11 (MT), DMU_17 (ES) \\
\hline DMU_3 (CE) & DMU_11 (MT), DMU_17 (ES) \\
\hline DMU_4 (PE) & DMU_11 (MT), DMU_13 (PB), DMU_17 (ES) \\
\hline DMU_5 (BA) & DMU_13 (PB), DMU_6 (MG) \\
\hline DMU_7 (RJ) & DMU_17 (ES), DMU_11 (MT) \\
\hline DMU_9 (PR) & DMU_13 (PB), DMU_6 (MG) \\
\hline DMU_10 (RS) & DMU_11 (MT) \\
\hline DMU_12 (GO/DF) & DMU_11 (MT), DMU_15 (MA), DMU_17 (ES) \\
\hline DMU_14 (RN) & DMU_17 (ES), DMU_11 (MT) \\
\hline DMU_16 (SC) & DMU_17 (ES), DMU_11 (MT) \\
\hline
\end{tabular}

Disponível em: https://www.nucleodoconhecimento.com.br/administracao/mensuracao-daeficiencia 


\begin{tabular}{l|l}
\hline DMU_18 (PI) & DMU_17 (ES), DMU_11 (MT) \\
\hline DMU_19 (MS) & DMU_13 (PB), DMU_6 (MG), DMU_15 (MA) \\
\hline DMU_22 (AC/RO) & DMU_11 (MT), DMU_15 (MA), DMU_17 (ES) \\
\hline DMU_23 (TO) & DMU_13 (PB), DMU_6 (MG)
\end{tabular}

Fonte: Dados da pesquisa

Percebe-se que a existência de ineficiências na maioria das superintendências, impede que o DNIT atinja a eficiência na execução orçamentária e financeira dos recursos destinados ao PAC para a manutenção da malha rodoviária federal de sua responsabilidade. Dessa forma, a eliminação das ineficiências relativas é fundamental para proporcionar maior nível de qualidade no oferecimento dos serviços de infraestrutura. Portanto, uma forma de reduzir tais disparidades é observar o que as superintendências eficientes estão fazendo e, então, segui-las.

\section{CONSIDERAÇÕES FINAIS}

A partir dos resultados apresentados pela Análise Envoltória de Dados (DEA) no modelo BCC orientado a outputs. (BCC-O) e considerando os objetivos desse trabalho, chegou-se as seguintes de que os resultados apresentados na tabela 05 evidenciam que apenas 9 das 23 Superintendências Regionais atingiram o índice de $100 \%$ de eficiência, ou seja, a fronteira padrão de eficiência é representada apenas pelas Superintendências Regionais dos estados de Minas Gerais, São Paulo, Mato Grosso, Paraíba, Maranhão, Espírito Santo, Alagoas, Sergipe e Rio de Janeiro. Dessa forma, constata-se que o DNIT foi ineficiente na execução orçamentária e financeira dos recursos destinados ao PAC para a manutenção da malha rodoviária de sua responsabilidade no período compreendido entre os anos de 2009 e 2015.

No entanto, é possível evidenciar, também na tabela 05 , que apenas 1 Superintendência não atingiu um índice superior a $90 \%$ de eficiência. Esses resultados permitem concluir que embora o DNIT tenha sido ineficiente, a tendência é que, em futuros exercícios financeiros, a autarquia se torne eficiente.

Disponível em: https://www.nucleodoconhecimento.com.br/administracao/mensuracao-daeficiencia 
A partir dos resultados obtidos com o software SIAD v.3.0, foi possível avaliar comparativamente a eficiência das Superintendências Regionais do DNIT na execução do orçamento autorizado para a manutenção da malha rodoviária federal. Dessa forma, foi possível identificar as Superintendências Regionais que apresentaram os melhores e piores níveis de eficiência na execução de recursos destinados ao PAC para a manutenção da malha rodoviária federal. Com base nesse ranking, é possível afirmar que os melhores níveis de eficiência foram apresentados pelas superintendências dos estados da Paraíba, Espirito Santo, Mato Grosso, Maranhão e Rio Grande do Sul, respectivamente. Enquanto os piores níveis de eficiência foram apresentados pelas superintendências dos estados do Paraná, Amazonas/Roraima e Bahia, respectivamente.

Portanto, a mensuração da eficiência do DNIT na execução dos recursos oriundos do PAC para a manutenção da malha rodoviária federal nos permite evidenciar o nível de eficiência dos gastos públicos realizados em Unidades Tomadoras de Decisão do DNIT e dessa forma, servir de base para que seus gestores tomem decisões no sentido de apresentarem resultados mais adequados às necessidades sociais.

\section{REFERÊNCIAS}

ARRETCHE, Marta T. S. Tendências no estudo sobre avaliação. RICO, Elizabeth Melo (org.). Avaliação de Políticas Sociais: Uma Questão em Debate. São Paulo, Cortez. 1998.

Decreto no 6.025, de 22 de janeiro de 2007. Instiui o Programa de Aceleração do Crescimento - PAC, o seu Comitê Gestor, e dá outras providências. Disponível em: http://www.planalto.gov.br/ccivil_03/_Ato20072010/2007/Decreto/D6025.htm.

. Balanços do PAC. Disponível em: http://www.brasil.gov.br/pac/. Acesso em 08/10/2019. 
- Presidência da República; Ministério do Planejamento, Orçamento e Gestão. PAC: Programa de Aceleração do Crescimento. Brasília, 2010. Disponível em: https://www.pac.gov.br/>. Acesso em: 25/09/2019.

CAMARGO, Francielle de O.; GUIMARÃES, Klicia M. S. O Princípio da Eficiência na Gestão Pública. Revista CEPPG- CESUC - Centro de Ensino Superior de Catalão, Ano XVI no 28. P.133-145, 1ํ Semestre/2013.

DA SILVA, Luiz Gustavo Inácio. A eficiência técnica do Exército Brasileiro consoante o aumento de recursos orçamentários. 109f. Dissertação (Mestrado). Universidade do Minho. Escola de Economia e Gestão, 2015.

DE LIMA, Hugo Maracajá Vaz; DINIZ, Josedilton Alves. Eficiência na aplicação dos recursos públicos pelos Tribunais de Justiça do Brasil. XX Congresso Brasileiro de Custos Uberlândia, MG. 2013.

FARIA, F. P; JANUZZI, P. M; SILVA, S. J. Eficiência dos gastos municipais em saúde e educação: uma investigação através da análise envoltória no Estado do Rio de Janeiro. Revista de Administração Pública - RAP. 42(1):155-177, JAN./FEV. Rio de Janeiro, 2008.

MEZA, Lidia Ângulo et al. SIADv3.0 - Sistema Integrado de Apoio à Decisão: uma implementação computacional de modelos de análise de envoltória de dados. Disponível em: <https://www.researchgate.net/publication/256373425_SIAD__Sistema_Integrado_de_Apoio_a_Decisao_uma_implementacao_computacional_de _modelos_de_analise_de_envoltoria_de_dados>. Acesso em 26/08/2019.

PALUDO, Augustinho Vicente, PROCOPIUCK, Mario. Planejamento Governamental: Referencial Teórico, Conceitual e Prático, $2^{\mathrm{a}}$ ed. São Paulo: Atlas, 2014. 
PEÑA, Carlos Rosano. Um Modelo de Avaliação da Eficiência da Administraçáo Pública através do Método Análise Envoltória de Dados (DEA). RAC - Revista de Administração Contemporânea, Curitiba. vol. 12, núm. 1, jan/mar, pp. 83-106. 2008.

PIMENTA, H.L.N.; SOARES DE MELLO, J.C.C.B. Modelo DEA-Savage para análise de eficiência do parque de refino brasileiro. Relatórios de Pesquisa em Engenharia de Produção da UFF, v. 5, p. 5, 2005.

\section{APÊNDICE - REFERÊNCIAS DE NOTA DE RODAPÉ}

2. http://www.orcamentofederal.gov.br/orcamentos-anuais

3. http://www.orcamentofederal.gov.br/orcamentos-anuais

Enviado: Dezembro, 2019.

Aprovado: Agosto, 2020. 